\title{
Non-union of a Plantar Calcaneal Spur Fracture: A case report
}

\author{
by Shishir P. Nawghare, D’Ortho ${ }^{1} \rrbracket$, Yogesh V. Kolwadkar , MS (Ortho), MRCS (Ed) ${ }^{2}$, Pradeep K. \\ Singh, MS Ortho ${ }^{3}$
}

The Foot \& Ankle Journal 1 (7): 2

We report a case of a 35-year-old female who presents with acute pain involving the heel after minimal trauma. This case represents a non union of a fractured plantar calcaneal spur. We present the clinical and radiological data on a single case of non-union of a fractured plantar calcaneal spur. Results revealed that a fracture of a plantar calcaneal spur can proceed to a non-union if neglected, but may still be managed conservatively. A thorough literature search and an exhaustive online search using various search engines revealed only two reported fractures of a plantar heel spur. This is an unusual fracture and only the third case reported in literature.

Key words: Infracalcaneal spur, heel spur

Accepted: May $2008 \quad$ Published: July 2008

This is an Open Access article distributed under the terms of the Creative Commons Attribution License. It permits unrestricted use, distribution, and reproduction in any medium, provided the original work is properly cited. (The Foot \& Ankle Journal (www.faoj.org)

\begin{abstract}
A bony prominence on the plantar aspect of the calcaneus at the attachment of the plantar aponeurosis is called a heel spur. Traction on the periosteum with consequent subperiosteal ossification may lead to its development. The patient complains of pain on the undersurface of heel on standing and walking and pain that is relieved by rest. It is characterized by localized tenderness and the discomfort may be increased by passive dorsiflexion of the ankle.
\end{abstract}

\footnotetext{
Address correspondence to: Dr. Shishir Nawghare D’Ortho Assistant Lecturer, Orthopaedics, DMIMS, Wardha

187, Surendra Nagar, Nagpur, Maharashtra, India 440015.

Email: shishirnawghare@yahoo.com

${ }^{1}$ Assistant Lecturer, Orthopaedics, DMIMS, Wardha. Surendra Nagar, Nagpur, Maharashtra, India 40015.

2 Assistant Professor Orthopaedics, DMIMS, Wardha. Surendra Nagar,

Nagpur, Maharashtra, India 40015.

3 Associate Professor Orthopaedics, DMIMS, Wardha. Surendra Nagar,

Nagpur, Maharashtra, India 40015.
}

Radiographs may or may not reveal a plantar calcaneal spur. Conservative treatments include bed rest, hot soaks, needling the tender area with local anesthetic, local injection of corticosteroids and wearing a sponge rubber heel pad. Surgery is reserved for resistant cases. ${ }^{1}$

A literature search and an exhaustive online search using various search engines indicated only two reported fractures of a plantar calcaneal heel spur. ${ }^{2,3}$ This is an unusual fracture and only the third case reported in literature. 


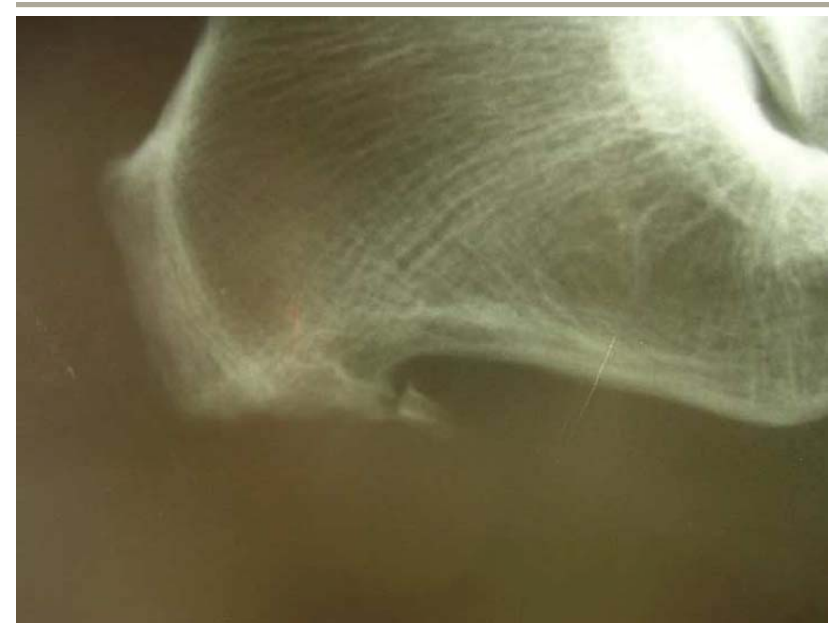

Figure 1 Radiograph of calcaneal spur fracture.

Two mechanisms of action can result in this type of fracture. The first involves direct impact on the inferior aspect of the calcaneal tuberosity. A sudden pull on the exostosis from the originating soft-tissue structures may also result in fracture. (Would include here the structures that attach to this area) The windlass effect may come into play as the plantar fascia and the flexor digitorum brevis may contribute to an avulsion of the plantar calcaneal spur. ${ }^{4,5,6,7}$

\section{Case Report}

We report the case of a 35 year-old lady who was attacked by a monkey in her courtyard. In an attempt to run she stamped her foot forcefully on the ground. She escaped unharmed, but the pain in her heel started bothering her. It was difficult for her to bear weight on the affected foot.

She presented twelve hours after the incident. On examination, there was no obvious swelling. There was tenderness at the plantar, central aspect of her heel. We could not elicit tenderness anywhere else. The range of motion at the ankle and the subtalar joint were full and painless. There was no neurovascular deficit.

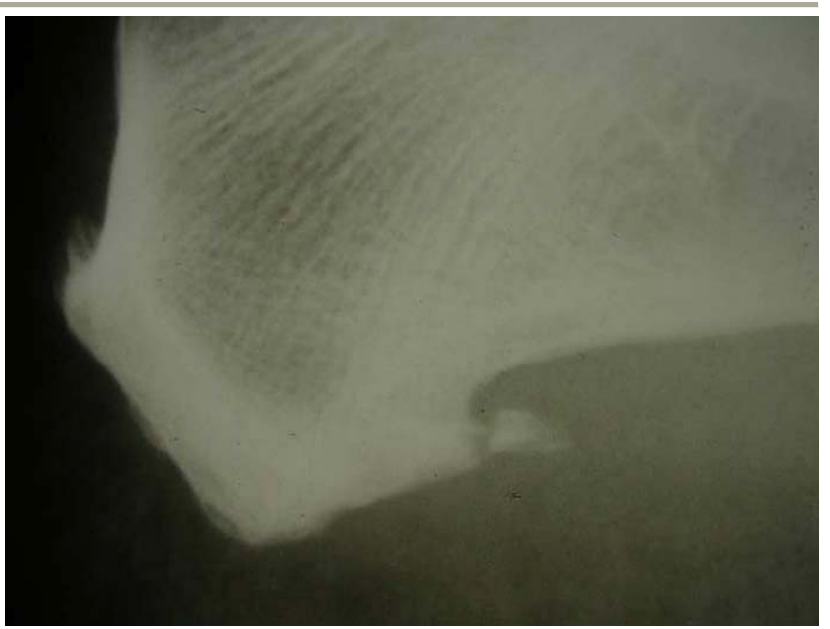

Figure 2 Calcaneal spur fracture at 7 months follow up.

Radiographs revealed an undisplaced fracture of an infracalcaneal spur. (Fig. 1) We advised antiinflammatory drugs and a below knee cast for 4 weeks. Unfortunately, the patient found the cast uncomfortable and removed it on her own after only four days. She did not follow-up initially with the hospital and continued walking. She reported to the hospital after seven months. On clinical examination, there was no local tenderness and she was comfortable while walking fullweight bearing. The radiograph showed displacement at the fracture site and no signs worrisome for non-union. (Fig. 2) As the patient had no complaints about her foot and was comfortable, we decided to withhold future treatment.

\section{Discussion}

A infracalcaneal heel spur is detected frequently on radiograph examination. It is a wellunderstood cause of plantar heel pain. Fracture of the infracalcaneal spur is rare. It is easily detected by radiographs and can be treated conservatively ${ }^{2}$ or if needed, surgically. ${ }^{3}$ On the other hand, if neglected, like any other fracture, it may progress to a non-union. 
In this case, the fracture occurred due to direct impact on the inferior aspect of the calcaneal tuberosity. As little evidence was available in the literature regarding its management we decided to treat the fracture conservatively. The patient was not compliant and continued bearing weight without any form of immobilization. The fracture displaced and progressed to non-union that was diagnosed at 7 months. Despite the progression to a non-union the patient was asymptomatic.

Based on our findings in this case study and a review of the brief literature, we recommend a conservative line of management for this type of fracture. Though rare, a possibility of fracture of an infracalcaneal spur should always be considered in all cases of heel pain.

All authors were fully involved in the study and preparation of the manuscript.

No benefits in any form have been received or will be received from a commercial party related directly or indirectly to the subject of this article. No funds were received in support of this report.

\section{References}

1. Turek SL. Orthopaedic Principles and their Application. Fourth edition, Volume 2:1472.

2. Burks JB ,Buk A.Bilateral fractures of the infracalcaneal exostosis. JFAS. Jan-Feb; 42(1):43-44, 2003

3. Subasi M, Kapukaya A, Kesemenli C, Coban V. Rare fractures. Ulus Travma Derg. 7:282-284, 2001.

4. Hicks JH. Mechanics of the foot I: the joints. J Anat. 87:345-357, 1953.

5. Hicks JH. Mechanics of the foot II: the plantar aponeurosis and the arch. J Anat. 88:25-31, 1954.

6. Hicks JH. The foot as a support. Acta Anat. 25:34-45, 1955.

7. Hicks JH. Mechanics of the foot IV: action of the muscles on the foot in standing. Acta Anat. 27:180-192, 1956. 